\title{
Testing the parton evolution with the use of two-body final states
}

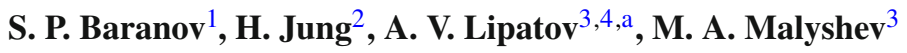 \\ ${ }^{1}$ P.N. Lebedev Physics Institute, Moscow 119991, Russia \\ 2 Deutsches Elektronen-Synchrotron, Notkestrasse 85, Hamburg, Germany \\ ${ }^{3}$ Skobeltsyn Institute of Nuclear Physics, Lomonosov Moscow State University, Moscow 119991, Russia \\ ${ }^{4}$ Joint Institute for Nuclear Research, Dubna 141980, Moscow Region, Russia
}

Received: 20 September 2016 / Accepted: 7 December 2016 / Published online: 27 December 2016

(C) The Author(s) 2016. This article is published with open access at Springerlink.com

\begin{abstract}
We consider the production of $b \bar{b}$ quarks and Drell-Yan lepton pairs under LHC conditions focusing attention on the total transverse momentum of the produced pair and on the azimuthal angle between the momenta of the outgoing particles. Plotting the corresponding distributions in bins of the final-state invariant mass, one can reconstruct the full map of the transverse momentum dependent parton densities in a proton. We give examples of how these distributions can look like at the LHC energies.
\end{abstract}

Experiments of new generation running at the LHC yield plenty of high precision data. In order to properly interpret these data we need the parton distribution functions to be known with adequately good accuracy. This, in turn, raises the question of a detailed measurement of the parton distributions. In this note we focus attention on two important kinematic observables which enable us to reconstruct the full map of the transverse momentum dependent (TMD), or unintegrated, parton densities. We address the LHC conditions ( $p p$ collisions at $\sqrt{s}=7 \mathrm{TeV}$ ), for which we give a number of illustrations.

The evolution of TMD gluon densities can be explored with the production of $b \bar{b}$ pairs. At the LHC energies, this process is dominated by the direct leading-order (LO) offshell gluon-gluon fusion subprocess

$g^{*}\left(k_{1}\right)+g^{*}\left(k_{2}\right) \rightarrow b\left(p_{1}\right)+\bar{b}\left(p_{2}\right)$,

while the contribution from the quark-antiquark annihilation is of almost no importance because of the comparatively low quark densities. The four-momenta of corresponding particles are given in the parentheses. The present calculation of the process (1) is fully identical to that performed previously [1]. The evolution of TMD quark densities can be explored with the production of Drell-Yan lepton pairs. This

a e-mail: lipatov@theory.sinp.msu.ru process is dominated by the off-shell quark-antiquark annihilation subprocess

$q^{*}\left(k_{1}\right)+\bar{q}^{*}\left(k_{2}\right) \rightarrow l^{+}\left(p_{1}\right)+l^{-}\left(p_{2}\right)$,

where $q$ is for the valence and sea quarks and $\bar{q}$ stands for the sea anti-quarks. The present calculation of the process (2) is fully identical to that from [2]. We do not consider here higher-order corrections $q+\bar{q} \rightarrow l^{+}+l^{-}+g$ since they are already taken into account in the $k_{T}$-factorization approach [3-7] as a part of the evolution of TMD quark densities.

The final states of the processes (1) and (2) are represented by two-body systems with fully reconstructible kinematics where the transverse momentum $p_{T}$ of the $b \bar{b}$ or lepton pair measures the net transverse momentum of the initial gluons or quarks, the invariant mass of the pair measures the product of longitudinal momentum fractions, $M^{2}=x_{1} x_{2} s$, and the rapidity of the pairs measures the ratio of the momentum fractions, $y=(1 / 2) \ln \left(x_{1} / x_{2}\right)$. A useful complementary observable is the difference between the azimuthal angles of produced particles $\Delta \phi$. In the LO of collinear QCD factorization, the $p_{T}$ and $\Delta \phi$ distributions degenerate into delta functions at $p_{T}=0$ and $\Delta \phi=0$, and the continuous spectra can only be obtained by including higher-order corrections. In the $k_{T}$-factorization approach, these radiative corrections are automatically taken into account in the form of TMD parton densities. Comparing the $p_{T}$ and $\Delta \phi$ spectra at varying gluon momentum fraction $x$ we watch the evolution of parton distributions.

To simulate the $b \bar{b}$ pair production we used the latest JH'2013 parametrization [8] for the TMD gluon densities in a proton. The input parameters of this gluon distribution were fitted to describe the proton structure function $F_{2}$. To simulate the production of Drell-Yan lepton pairs we applied complementary TMD valence quark distributions from the same set [8]. The necessary TMD sea quark densities are calculated from the gluon ones in the approximation 

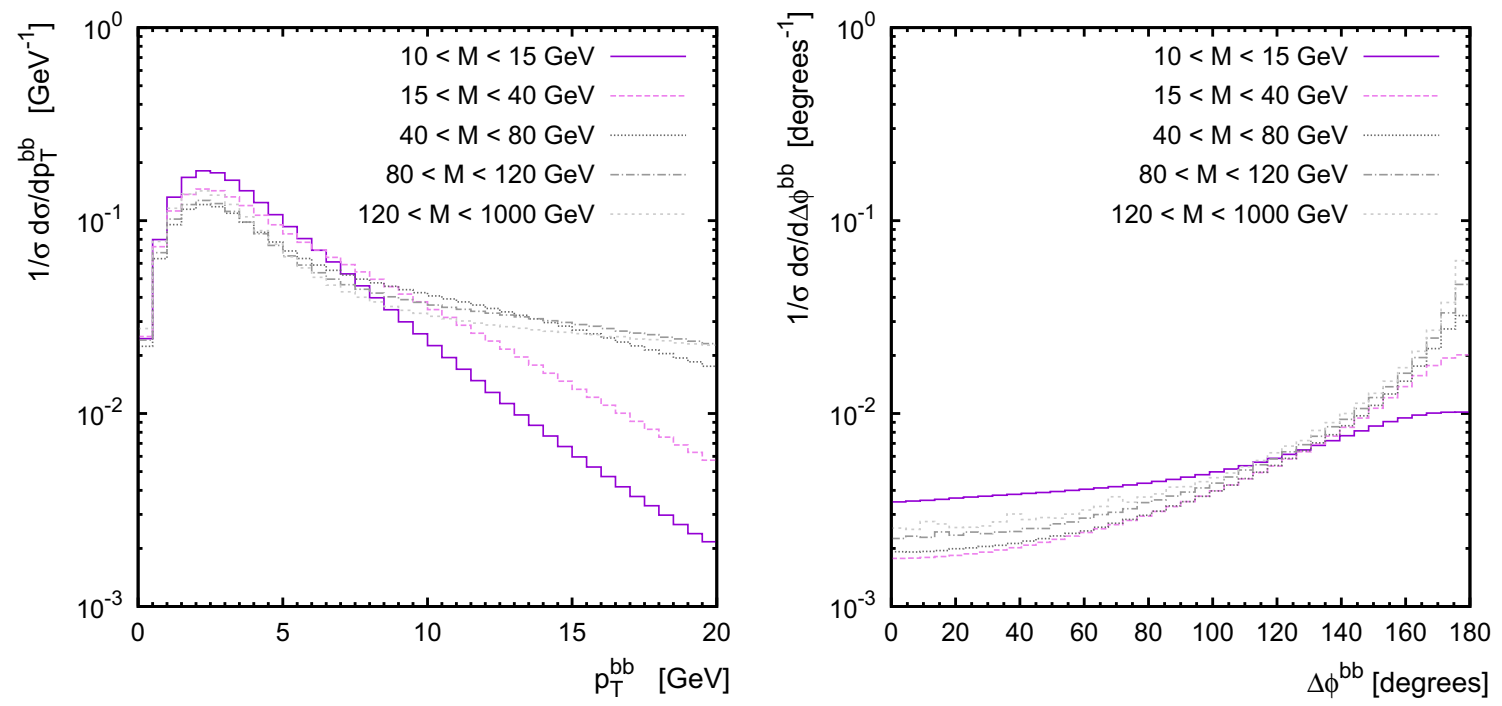

Fig. 1 Spectra of the $b \bar{b}$ pair transverse momentum $p_{T}$ and the azimuthal angle between the beauty quarks $\Delta \phi$ for several different intervals of the $b \bar{b}$ invariant mass $M$
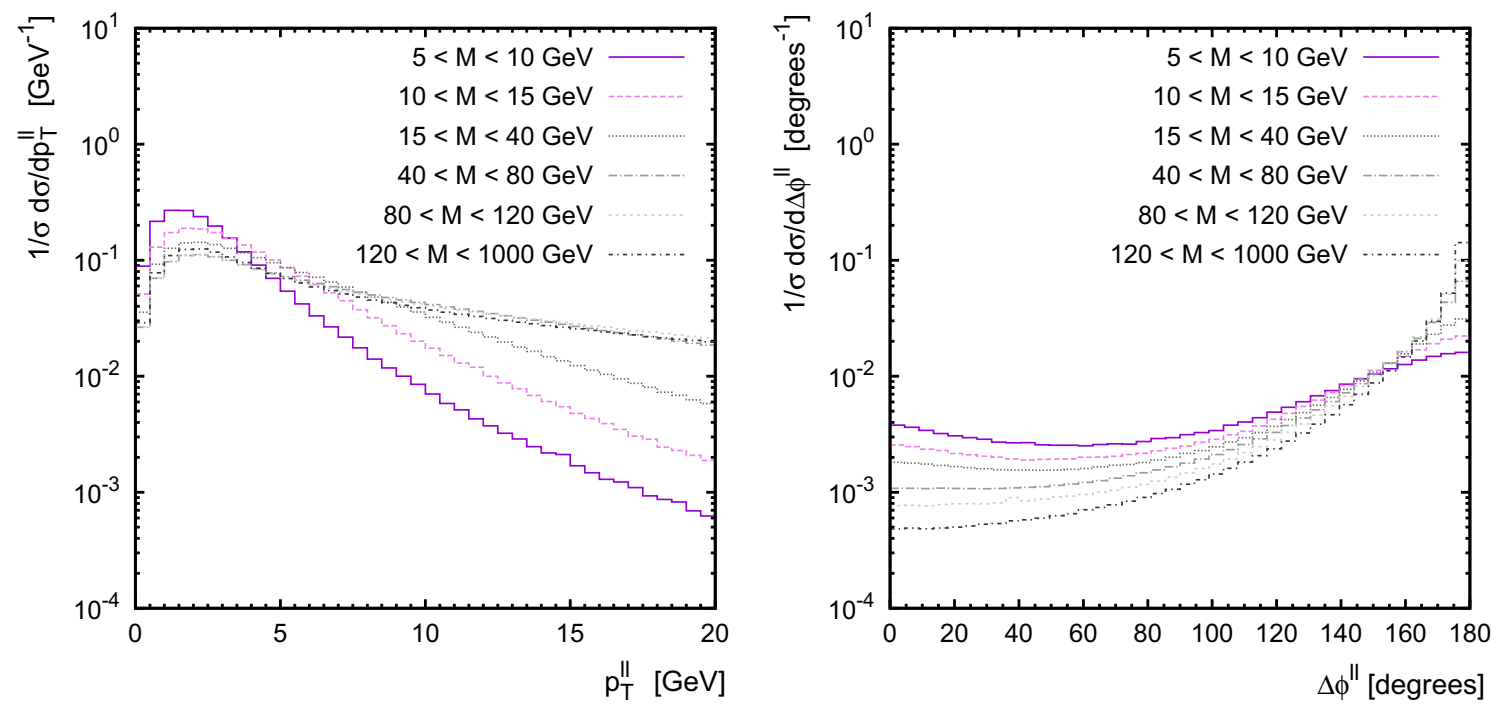

Fig. 2 Spectra of the Drell-Yan lepton pair transverse momentum $p_{T}$ and the azimuthal angle between the produced leptons $\Delta \phi$ for several different intervals of the dilepton invariant mass $M$

where the sea quarks occur in the last gluon-to-quark splitting [9].

The results of our calculations are displayed in Figs. 1, 2, 3, 4, 5, 6, and 7. Shown in Figs. 1 and 2 are the spectra of $b \bar{b}$ pair and dilepton transverse momentum $p_{T}$ and the azimuthal angle $\Delta \phi$ plotted for several different intervals of their invariant mass $M$. Here, to make the changes in shape more easily recognizable, we show the normalized differential cross sections. We see that with increasing $M$ the maximum in the $p_{T}$ spectrum shifts gradually to higher values, and the whole distribution becomes more flat. The $\Delta \phi$ distribution moves toward $\Delta \phi \simeq \pi$, which is due to the inequality $M \gg p_{T}$.
The latter becomes even stronger at high $M$ (see Fig. 3). As one can see from Fig. 2, quark distributions follow the same trend as gluon densities.

The observed behavior of the calculated $p_{T}$ and $\Delta \phi$ distributions is related to the different regions of $x$ and/or parton transverse momenta probed in the considered $M$ bins. In fact, with increasing $M$, the $x$ values obtained shifted toward unity, irrespectively of the rapidities of final-state particles, as is demonstrated in Figs. 4 and 5. The latter results show decreasing of the average parton transverse momentum generated in the non-collinear parton evolution. At the highest $M$ bin, this average parton transverse momentum becomes 

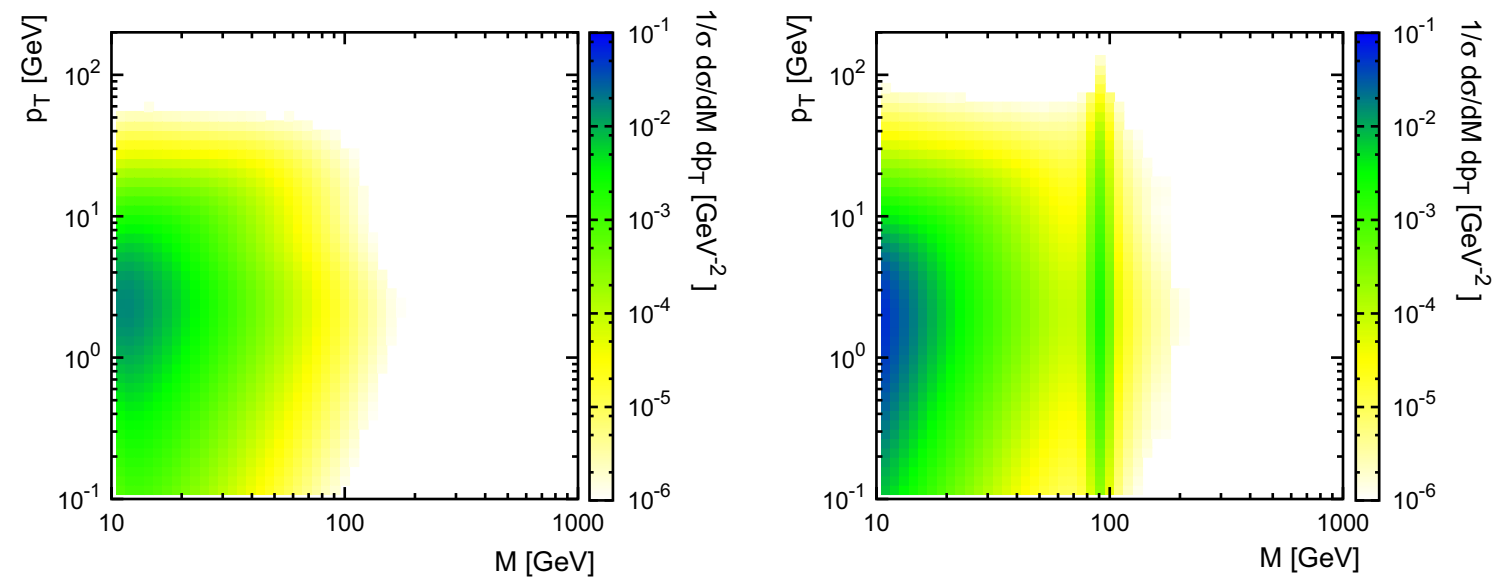

Fig. 3 Double differential cross sections of the $b \bar{b}$ (left panel) and Drell-Yan lepton pair production (right panel) as a functions of invariant mass $M$ and $p_{T}$ of the produced pair
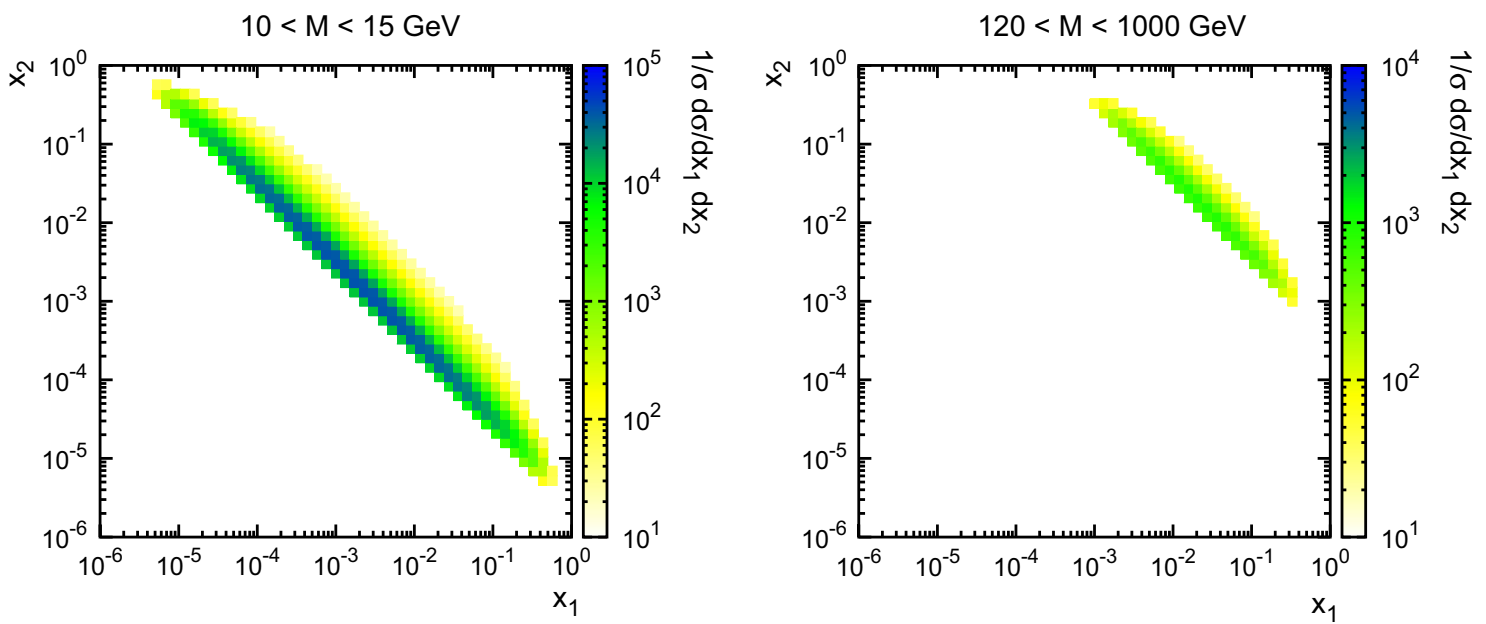

Fig. 4 Double differential cross sections of the $b \bar{b}$ pair production as a functions of $x_{1}$ and $x_{2}$ for two intervals of the $b \bar{b}$ invariant mass $M$
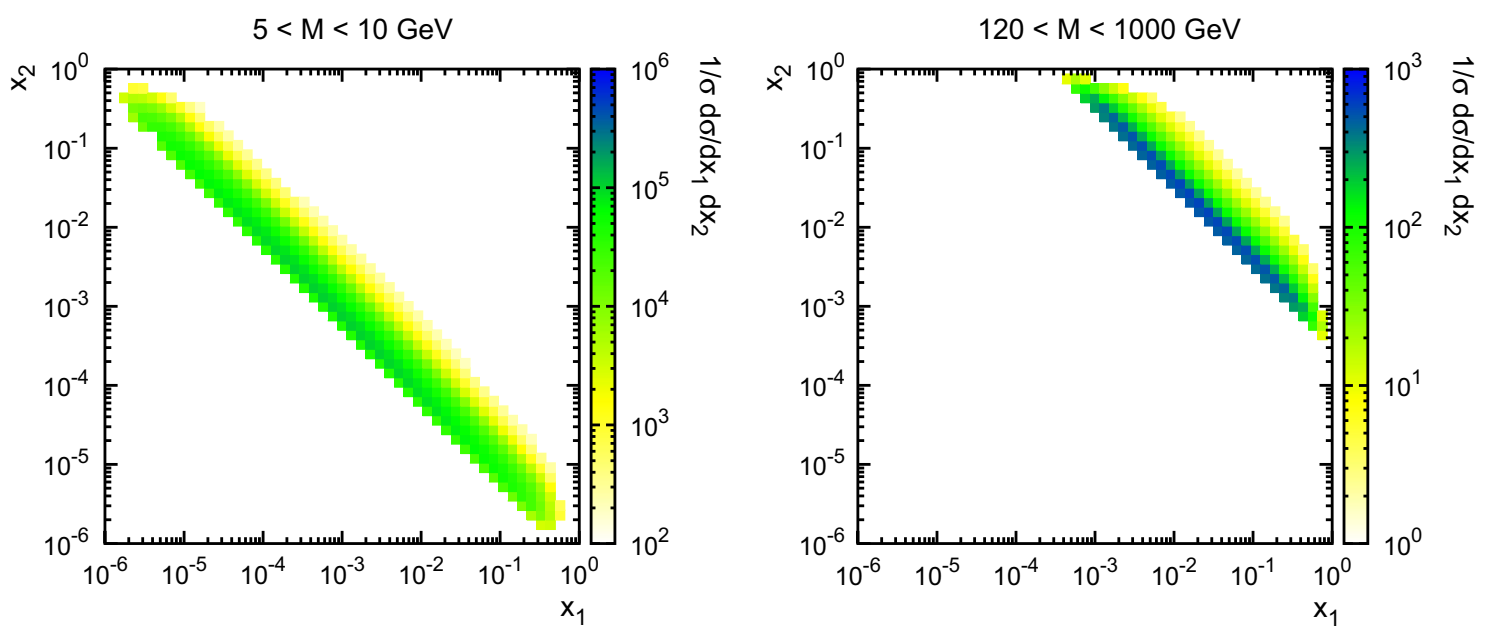

Fig. 5 Double differential cross sections of the Drell-Yan lepton pair production as a functions of $x_{1}$ and $x_{2}$ for two intervals of the dilepton invariant mass $M$ 

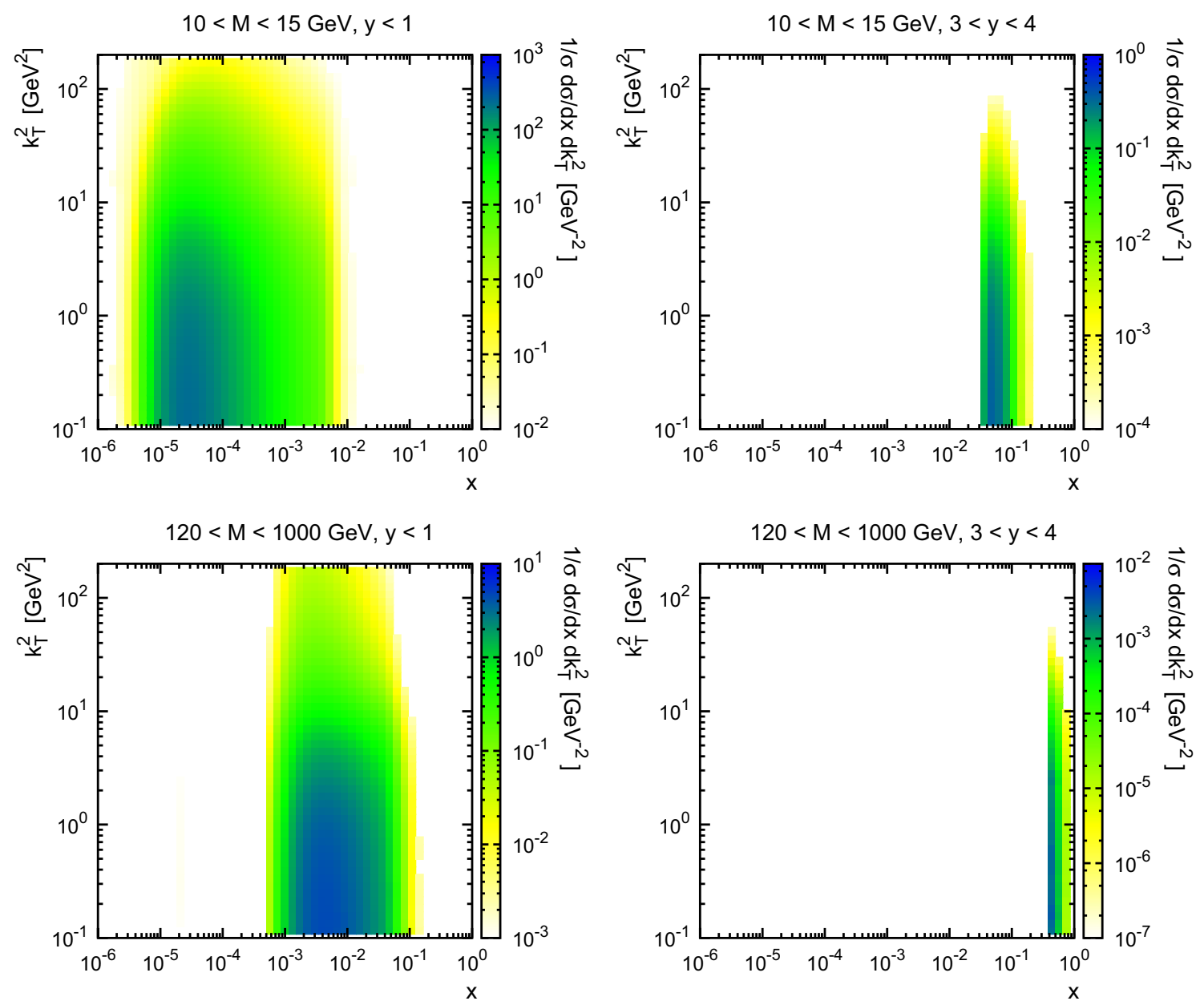

Fig. 6 Double differential spectra of the $b \bar{b}$ pair production as a function of $x$ and $\mathbf{k}_{T}^{2}$ for several different intervals of the $b \bar{b}$ invariant mass $M$ and rapidity $y$

small compared to the hard scale (which is order of $M$ ), so that the collinear kinematics of the partonic subprocesses is reproduced.

Besides the restrictions on the invariant mass, the special kinematical cuts on the final state give us further possibilities to find the region of $x$ and/or partonic transverse momenta we desire. It is illustrated in Figs. 6 and 7, where we plot the normalized differential cross sections of the considered subprocesses calculated as functions of $x$ and $\mathbf{k}_{T}^{2}$ (the longitudinal momentum fraction and transverse momentum of one of the colliding partons) with the additional cuts applied to the rapidity $y$ of the final-state quark or lepton pair. As an example, we used $y<1$ and $3<y<4$. We show that under these cuts one can probe different $x$ and/or $\mathbf{k}_{T}^{2}$ regions and extract information on the TMD parton distributions at the scale given by $M$. Note that the different $\mathbf{k}_{T}^{2}$ regions can be obtained under additional restrictions on the quark or lepton pair transverse momentum $p_{T}$ and/or azimuthal angle $\Delta \phi$.

The TMD parton distributions in a proton can be calculated using the different approaches (see, for example, [10] and the references therein). Of course, their different behavior as a function of $x$ and/or $\mathbf{k}_{T}^{2}$ is reflected in the description of the LHC data. For example, we compared the rapidity distribution of Drell-Yan lepton pair production calculated regarding the kinematical conditions imposed by the LHCb Collaboration [11] using the JH'2013 parton densities (as above) and the ones obtained from the KimberMartin-Ryskin (KMR) prescription [12,13] (see Fig. 8, left panel). The latter is a formalism to construct the TMD parton densities from the known conventional parton distributions. The key assumption is that the $k_{T}$ dependence enters at the last evolution step, so that usual DGLAP evolution [1417] can be used up to this step. The rapidity distribution of Drell-Yan pair production is sensitive to the $x$-behavior of TMD partons. One can clearly see that the difference between the TMD parton densities applied (Fig. 8, right panel) leads to a different description of recent $\mathrm{LHCb}$ data [11]. Therefore, the LHC experimental data for the processes considered can be used to constrain the TMD parton distributions in a proton. 

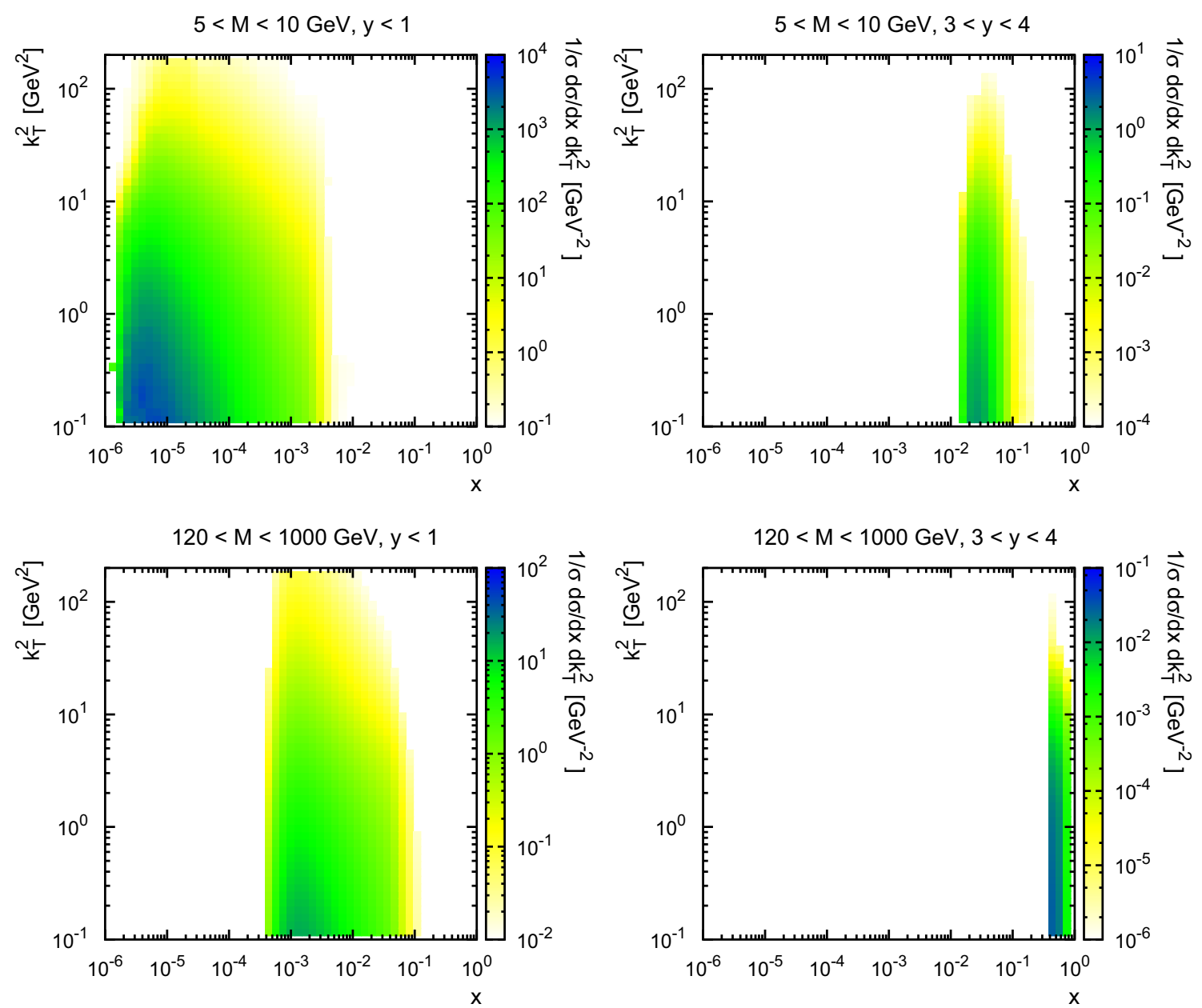

Fig. 7 Double differential spectra of the Drell-Yan pair production as a function of $x$ and $\mathbf{k}_{T}^{2}$ for several different intervals of the dilepton invariant mass $M$ and rapidity $y$

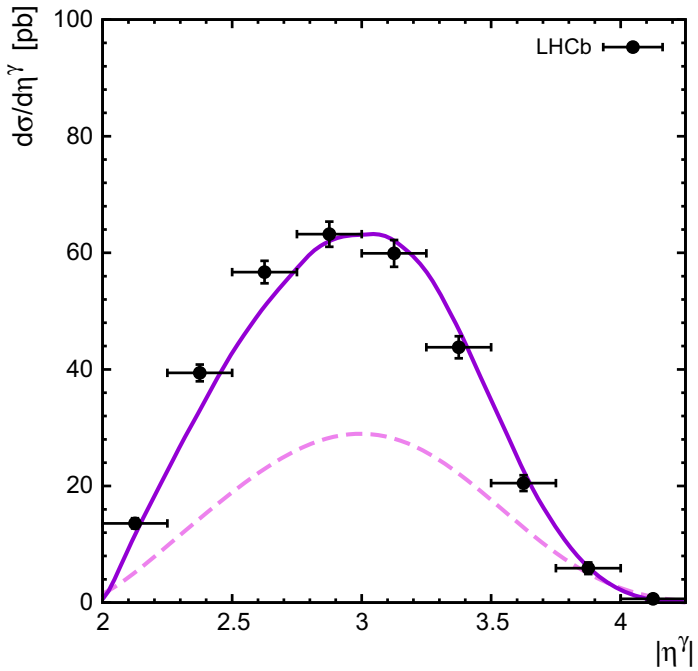

Fig. 8 Left panel the rapidity distribution of Drell-Yan lepton pair production at the LHC calculated at $60<M<120 \mathrm{GeV}$ and $\sqrt{s}=7 \mathrm{TeV}$. The cuts $p_{T}>20 \mathrm{GeV}$ and $2<\eta<4.5$ are applied for both leptons. The experimental data are from LHCb [11]. Right panel the TMD up

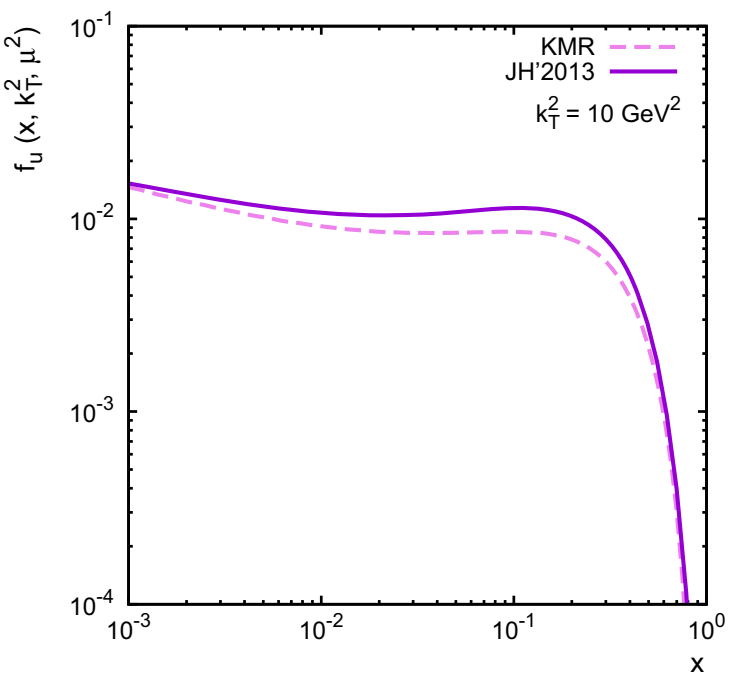

quark distributions calculated as a function of $x$ at $\mathbf{k}_{T}^{2}=10 \mathrm{GeV}$ and $\mu^{2}=100 \mathrm{GeV}^{2}$. The solid and dashed curves on both panels correspond to the JH'2013 and KMR sets, respectively 
Additionally, we investigate the dependence of estimates presented above on the parton shower effects using the Monte Carlo event generator CASCADE [18]. As expected, we observe only a very small contribution of the initial-state parton shower, since in the $k_{T}$-factorization approach it does not influence the transverse momentum of the gluons (because it is determined from the TMD gluon density).

Thus, we conclude that one can map the evolution of parton distributions at the scale $M$ from high values of proton longitudinal momentum fraction $x$ to low ones by applying different cuts on the final states. This is important for further precise determination of the TMD quark and gluon densities in a proton from the LHC data.

Acknowledgements This research was supported in part by RFBR Grant 16-32-00176-mol-a and grant of the President of Russian Federation NS-7989.2016.2. We are grateful to DESY Directorate for the support in the framework of Moscow - DESY project on Monte-Carlo implementation for HERA - LHC. We are also grateful to Nikolai Zotov for all his enthusiasm, the many discussions and the very good time we had together. N. Zotov passed away in January 2016; we will miss him.

Open Access This article is distributed under the terms of the Creative Commons Attribution 4.0 International License (http://creativecomm ons.org/licenses/by/4.0/), which permits unrestricted use, distribution, and reproduction in any medium, provided you give appropriate credit to the original author(s) and the source, provide a link to the Creative Commons license, and indicate if changes were made.

Funded by SCOAP ${ }^{3}$.

\section{References}

1. H. Jung, M. Krämer, A.V. Lipatov, N.P. Zotov, Phys. Rev. D 85, 034035 (2012)

2. S.P. Baranov, A.V. Lipatov, N.P. Zotov, Phys. Rev. D 89, 094025 (2014)

3. L.V. Gribov, E.M. Levin, M.G. Ryskin, Phys. Rep. 100, 1 (1983)

4. E.M. Levin, M.G. Ryskin, Phys. Rep. 189, 268 (1990)

5. S. Catani, M. Ciafaloni, F. Hautmann, Phys. Lett. B 242, 97 (1990)

6. S. Catani, M. Ciafaloni, F. Hautmann, Nucl. Phys. B 366, 135 (1991)

7. J.C. Collins, R.K. Ellis, Nucl. Phys. B 360, 3 (1991)

8. H. Jung, F. Hautmann, Nucl. Phys. B 883, 1 (2014)

9. F. Hautmann, M. Hentschinski, H. Jung, arXiv:1205.1759 [hep-ph]

10. F. Hautmann, H. Jung, M. Krämer, P.J. Mulders, E.R. Nocera, T.C. Rogers, A. Signori, Eur. Phys. J. C 74, 3220 (2014)

11. LHCb Collaboration, JHEP 02, 106 (2013)

12. M.A. Kimber, A.D. Martin, M.G. Ryskin, Phys. Rev. D 63, 114027 (2001)

13. G. Watt, A.D. Martin, M.G. Ryskin, Eur. Phys. J. C 31, 73 (2003)

14. V.N. Gribov, L.N. Lipatov, Sov. J. Nucl. Phys. 15, 438 (1972)

15. L.N. Lipatov, Sov. J. Nucl. Phys. 20, 94 (1975)

16. G. Altarelli, G. Parisi, Nucl. Phys. B 126, 298 (1977)

17. Yu.L. Dokshitzer, Sov. Phys. JETP 46, 641 (1977)

18. H. Jung et al., Eur. Phys. J. C 70, 1237 (2010) 